\title{
Fine-Tuning Roles of Osa-miR159a in Rice Immunity Against Magnaporthe oryzae and Development
}

\author{
Jin-Feng Chen ${ }^{\dagger}$, Zhi-Xue Zhao ${ }^{\dagger}$, Yan Li ${ }^{\dagger}$, Ting-Ting Li, Yong Zhu, Xue-Mei Yang, Shi-Xin Zhou, He Wang, \\ Ji-Qun Zhao, Mei Pu, Hui Feng, Jing Fan, Ji-Wei Zhang, Yan-Yan Huang and Wen-Ming Wang (D)
}

\begin{abstract}
Background: Rice blast caused by Magnaporthe oryzae is one of the most destructive diseases of rice. An increasing number of microRNAs (miRNAs) have been reported to fine-tune rice immunity against $M$. oryzae and coordinate with growth and development.

Results: Here, we showed that rice microRNA159a (Osa-miR159a) played a positive role in rice resistance to M. oryzae. The expression of Osa-miR159a was suppressed in a susceptible accession at 12, 24, and $48 \mathrm{~h}$ postinoculation (hpi); it was upregulated in a resistant accession of $M$. oryzae at $24 \mathrm{hpi}$. The transgenic rice lines overexpressing Osa-miR159a were highly resistant to M. oryzae. In contrast, the transgenic lines expressing a short tandem target mimic (STTM) to block Osa-miR159a showed enhanced susceptibility. Knockout mutations of the target genes of Osa-miR159a, including OsGAMYB, OsGAMYBL, and OsZF, led to resistance to M. oryzae. Alteration of the expression of Osa-miR159a impacted yield traits including pollen and grain development.

Conclusions: Our results indicated that Osa-miR159a positively regulated rice immunity against $M$. oryzae by downregulating its target genes. Proper expression of Osa-miR159a was critical for coordinating rice blast resistance with grain development.
\end{abstract}

Keywords: microRNA, Osa-miR159a, Short tandem target mimic, Magnaporthe oryzae, Rice blast disease

\section{Background}

Plant microRNAs (miRNAs) act as fine-tuning regulators and play regulatory roles in gene expression via cleavage, translational inhibition, or DNA methylation of target sites with sequences complementary to the miRNAs (Song et al. 2019). To date, more than 38,000 mature miRNAs have been reported in miRBase (http:// www.mirbase.org/). Among them, 757 mature miRNAs have been identified in rice.

miRNAs play major roles in many biological processes, including functions related to response to biotic and abiotic stressors (Jones-Rhoades et al. 2006; Miura et al.

\footnotetext{
* Correspondence: j316wenmingwang@163.com

†Jin-Feng Chen, Zhi-Xue Zhao and Yan Li contributed equally to this work. Rice Research Institute and Key Lab for Major Crop Diseases, Sichuan Agricultural University, Chengdu 611130, China
}

2010; Yan et al. 2016; Li et al. 2019b). Functional studies of many miRNAs have been done on Arabidopsis and rice. For example, knockout of miR396ef results in increased grain yield in rice via increasing grain size and panicle branching due to disinhibition of the expression of OsGRF4 and OsGRF6, which are the target genes of miR396 (Zhang et al. 2019; Miao et al. 2020). The overexpression of miR1873 results in defects in yield traits by repressing its target gene LOC_Os05g01790 (Zhou et al. 2020). miR535 is highly expressed in rice panicles. Enhanced accumulation of miR535 reduces plant height, modifies panicle architecture, and increases the grain length by regulating OsSPLs (Sun et al. 2019). miR167 regulates stamen and gynoecium development in immature flowers by regulating the target genes ARF6 and ARF8 in Arabidopsis (Wu et al. 2006). Increasing 
evidence shows that miRNAs are involved in rice immunity against Magnaporthe oryzae. For example, overexpression of miR1873 enhanced the susceptibility of rice to $M$. oryzae by regulating its target gene $L O C_{-}$ Os05g01790 (Zhou et al. 2020). In addition, miR396, miR169, miR164a, miR319b, and miR167d negatively regulate immunity against $M$. oryzae in rice ( $\mathrm{Li}$ et al. 2017a; Wang et al. 2018; Zhang et al. 2018; Chandran et al. 2019; Zhao et al. 2019), whereas miR398b, miR160a, miR166k-miR166h, miR7695, and miR162a positively regulate the response against $M$. oryzae in rice (Achard et al. 2004; Salvador-Guirao et al. 2018; Li et al. 2019a; Li et al. 2019b; Quoc et al. 2019; Li et al. 2020a).

The highly conserved and abundant 21 nucleotide (nt) miRNAs, miR159 and miR319, share a sequence identity of 17 out of $21 \mathrm{nt}$ matching with that of Arabidopsis (Palatnik et al. 2007). However, miR159 and miR319 function differently through distinct target genes. miR319 targets PROLIFERATING CELL NUCLEAR ANTIGEN BINDING FACTOR (TCP) transcription factor genes, which control leaf shape (Schwab et al. 2005; Palatnik et al. 2007), while miR159 targets a family of genes encoding R2R3 MYB transcription factors, which are referred to as GAMYBs or GAMYB-likes (GAMYBLs), and function in flowering and male fertility (Millar et al. 2019). The miR159-GAMYB regulatory module has been identified in major land plants, including Arabidopsis and rice. This module has been reported to act in growth and development. For example, in Arabidopsis, miR159 suppresses the expression of MYB33 and MYB65 to regulate plant growth and development. mir159a mir159b (mir159ab) double mutant displays severe growth and developmental defects including curled rosette leaves and stunted plant height (Allen et al. 2007). These phenotypes may be due to the failure to suppress the expression of MYB33 and MYB65 by miR159 (Allen et al. 2007; Alonso-Peral et al. 2010). Also, up-regulation of miR159 impacts anther development and delays flowering. Moreover, miR159 plays a crucial role in pollen fertility, and pollen-carried miR159 abolishes the expression of MYB33 and MYB65 in the central cell after fertilization, promoting endosperm nuclear division and seed development (Zhao et al. 2018). miR159 regulates flowering time and development during the short-day photoperiod by directly cleaving the mRNA of GAMYB-related genes that encode proteins involved in GA-promoted activation of LEAFY (Achard et al. 2004). In addition, miR159-MYB33 functions as a modifier of vegetative phase change in Arabidopsis (Guo et al. 2017).

The rice genome contains six Osa-miR159 genes generating five mature isoforms: Osa-miR159a, OsamiR159b, Osa-miR159c, Osa-miR159d, Osa-miR159e, and Osa-miR159f. These isoforms mediate mRNA cleavage of three genes, GAMYB (LOC_Os01g59660), GAMYBL (LOC_OsO6g40330), and ZF (encoding a C3HC4-type domain-containing zinc finger protein, $L O C_{-} O s 10 g 05230$ ). GAMYB has been shown to function in rice development. For example, miR159-GAMYB modulates the expression of gibberellic acid (GA)/abscisic acid (ABA)-related genes to maintain the energy supply and enhance developmental processes in Wuxiang $\mathrm{S}$, a photo-thermosensitive genic male sterile rice line (Zhang et al. 2016a). In addition, a few studies show that miR159 functions in plant immunity. For example, in cotton, miR159 and miR166 are increased in response to infection by Verticillium dahliae, and are exported to the fungal hyphae to silence the target genes $\mathrm{Ca}^{2+}$. dependent cysteine protease (Clp-1) and an isotrichodermin C-15 hydroxylase ( $\mathrm{HiC}-15)$, which are essential for fungal virulence (Zhang et al. 2016b). In Lilium regale, Ire-miR159a positively regulates the plant's resistance to grey mold caused by Botrytis elliptica by repressing the expression of its target gene $\operatorname{LrGAMYB}$ (Gao et al. 2020). In a previous study, we found that Osa-miR159a was differentially accumulated in susceptible and resistant accessions of rice ( $\mathrm{Li}$ et al. 2014). However, its function in rice immunity has not been characterized.

In this study, we further functionally characterized Osa-miR159a. To accomplish this, we obtained the transgenic lines overexpressing Osa-miR159a (OX159) and the suppressed expression of Osa-miR159a (STTM159) through short tandem target mimic (STTM), which is an effective method to block mature miRNA binding to target sites of the target genes (Yan et al. 2012). In addition, we also constructed the knockout transgenic lines of GAMYB, GAMYBL, and $Z F$ using the CRISPR/Cas9 method. Then, these transgenic lines were subjected to a $M$. oryzae disease assay and the phenotypic assay. We found that Osa-miR159a acts as a positive regulator in rice resistance to $M$. oryzae by suppressing $G A M Y B, G A M Y B L$, and $Z F$. It also impacted reproductive development in rice. Therefore, proper accumulation of Osa-miR159a was necessary to fine-tune the development and resistance to $M$. oryzae in rice.

\section{Results}

\section{Osa-miR159a is Responsive to M. oryzae Infection}

Previously, the expression of Osa-miR159a was reported to be responsive to $M$. oryzae or its elicitors ( $\mathrm{Li}$ et al. 2014; Li et al. 2016b; Li et al. 2019b). To confirm this conclusion, we examined its expression pattern in susceptible and resistant accessions of rice after inoculation of $M$. oryzae at the three-leaf seedling stage. The universally susceptible accession Lijiangxin Tuan Heigu (LTH) showed a severe disease phenotype, whereas the accession (IRBLkm-Ts) that contains the gene Pikm, which confers $M$. oryzae resistance displayed resistance (Fig. 1a). 

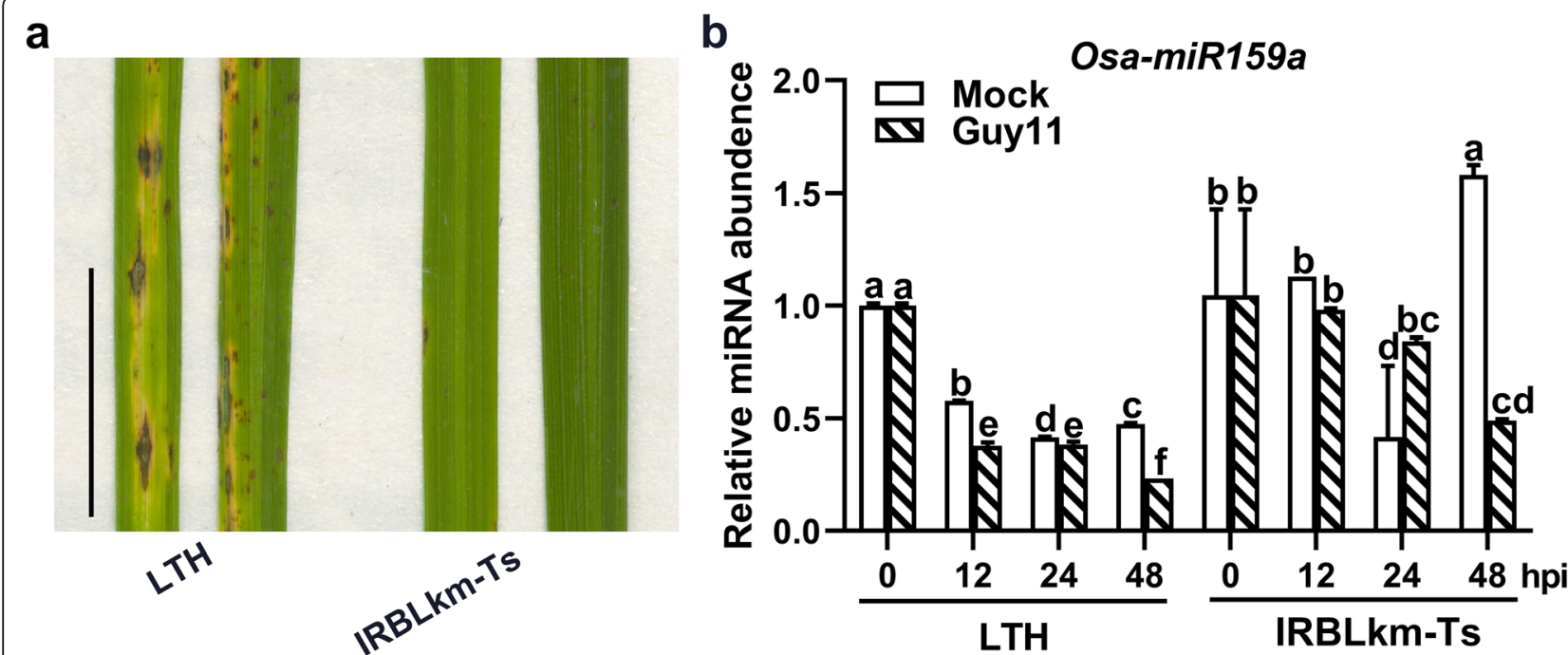

Fig. 1 Osa-miR159a is differentially responsive to Magnaporthe oryzae in the susceptible and resistant accessions. a Representative leaves show the blast disease phenotype at 5 days post-inoculation (dpi) with M. oryzae strain Guy11 in LTH and IRBLkm-Ts. Scale bars, $10 \mathrm{~mm}$. b Reverse transcription-quantitative PCR (RT-qPCR) shows the accumulation of Osa-miR159a in LTH and IRBLkm-Ts upon M. oryzae or mock treatment at the indicated time points. Error bars indicate SD $(n=3)$. Different letters above bars indicate significant differences $(P<0.05)$ as determined by a oneway ANOVA followed by Tukey's HSD post hoc test. This experiment was repeated two times with similar results

Compared with mock inoculation, M. oryzae infection resulted in decreased accumulation of Osa-miR159a at 12, 24, and $48 \mathrm{~h}$ post-inoculation (hpi) in LTH (Fig. 1b). In contrast, Osa-miR159a was significantly upregulated at $24 \mathrm{hpi}$ in IRBLkm-Ts (Fig. 1b), although it was also significantly decreased at $48 \mathrm{hpi}$. These data indicated that the response of Osa-miR159a to M. oryzae infection was different in the susceptible and resistant accessions. Therefore, Osa-miR159a may play a role in rice immunity against $M$. oryzae.

\section{Osa-miR159a Positively Regulates Rice Resistance to $M$. oryzae}

To explore how Osa-miR159a acts in the interaction between rice and $M$. oryzae, we made a construct overexpressing Osa-miR159a (OX159) and introduced the construct into Nipponbare (NPB), generating 24 independent transgenic lines, out of which we chose two lines that showed high Osa-miR159a accumulation for further investigation (Fig. 2a). We made a construct expressing STTM of Osa-miR159a (STTM159) and introduced it into NPB, which may prevent Osa-miR159a from binding to its target sites (Franco-Zorrilla et al. 2007; Todesco et al. 2010). We also selected two independent transgenic lines that showed a significant reduction of Osa-miR159a accumulation for further investigation (Fig. 2a). Then we conducted blast disease assays by punch- or spray- inoculation of the M. oryzae strain GZ8. We found that OX159 lines generated significantly smaller disease lesions than NPB harboring an empty vector (EV) (Fig. 2b and Fig. S1a). Consistently, the lesions from OX159 lines contained significantly less fungal mass and shorter lesion length than the control at 5 days post-inoculation (dpi) (Fig. 2c, d and Fig. S1b). In contrast, STTM159 lines generated significantly larger disease lesions than that of the control (Fig. 2e and Fig. S2a), and the lesions from STTM159 lines contained significantly more fungal mass and longer lesions than the control at 5 dpi (Fig. 2f, g and Fig. S2b, c). These data indicated that Osa-miR159a positively regulated the resistance of rice to M. oryzae.

Next, we exploited the GFP-tagged strain GZ8 to observe the infection process in sheath cells using laser scanning confocal microscopy. Compared with the control, our observation found that the infection progress was delayed in OX159 (Fig. S1c, d), but accelerated in STTM159 (Fig. S2d, e). At 24 hpi and 36 hpi, the percentages of invasive hyphae were much lower in OX159 (Fig. S1c, d) compared with the control; however, the percentages of invasive hyphae were greater in STTM159 (Fig. S2d, e). These results indicated that overexpressing Osa-miR159a delayed infection, whereas blocking Osa-miR159a facilitated M. oryzae infection.

To explain why Osa-miR159a positively regulated resistance to $M$. oryzae, we used RT-qPCR to examine the expressions of some marker genes, including OsNAC4, OsPR10b (Pathogenesis-Related 1b) and OSJAMYB, acting in immune responses after infection with $M$. oryzae (Park et al. 2012; Pan et al. 2014). The expression of OsNAC4 was higher in OX159 than in the control at 6 and $12 \mathrm{hpi}$ (Fig. S1e), whereas it was lower in STTM159 than in the control at 6 and 24 hpi (Fig. S2f). The expression of OsPR10b was higher in OX159 than in the control at 6 

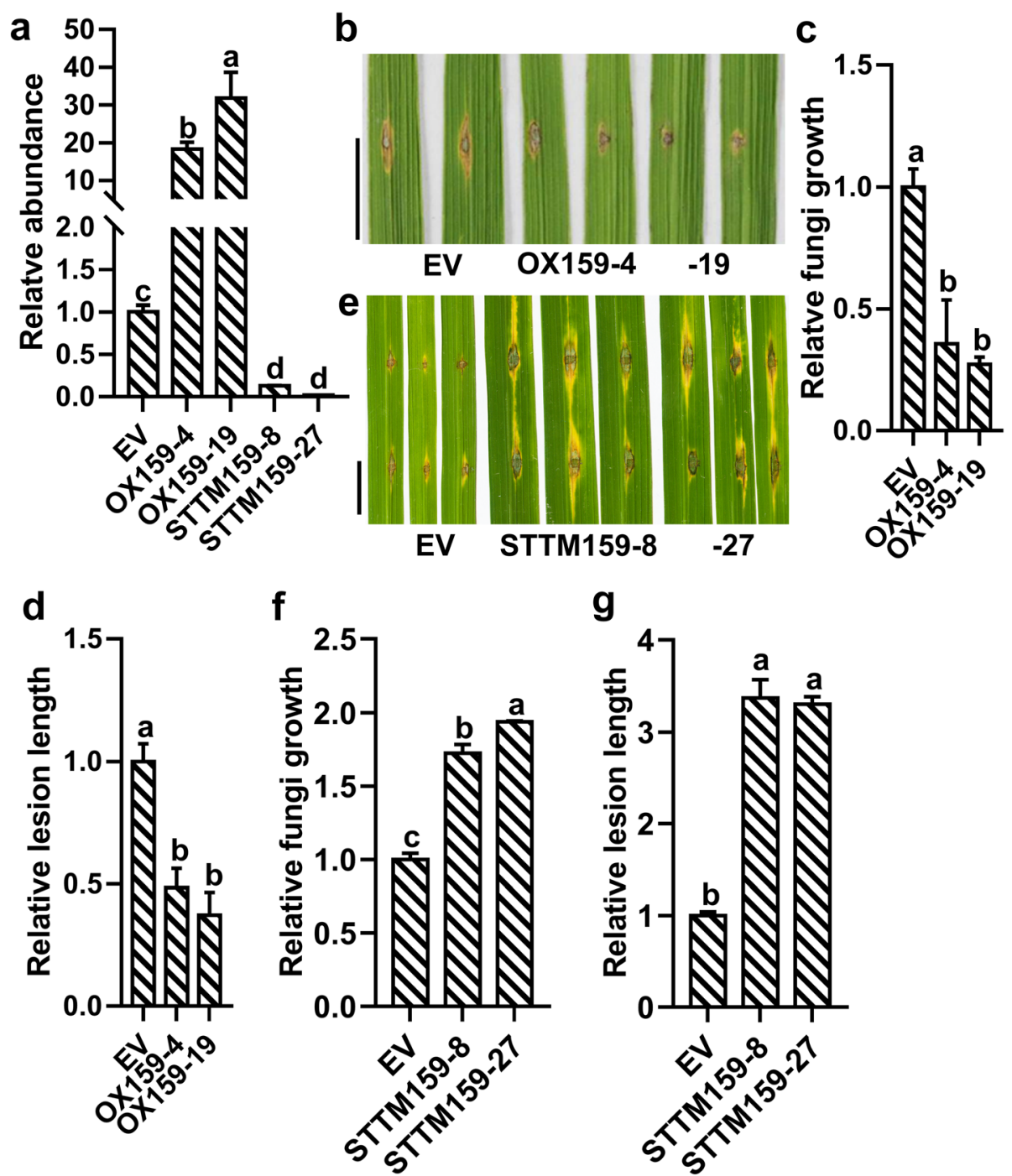

Fig. 2 Osa-miR159a alleviates rice susceptibility to Magnaporthe oryzae. a Reverse transcription-quantitative PCR (RT-qPCR) data shows the relative abundance of Osa-miR159a in transgenic lines containing 35S: MIR159a (OX159) or transgenic lines containing STTM159 in comparison with Nipponbare (NPB) containing empty vector (EV). b, e Blast disease phenotype at 5 days post-inoculation (dpi) of M. oryzae strain GZ8 by punchinoculation in the indicated lines. Scale bars, $10 \mathrm{~mm}$. $\mathbf{c}, \mathbf{f}$ Quantification analysis of $M$. oryzae biomass in (b) and (e), respectively. Error bars indicate SD $(n=3)$. Different letters above bars indicate significant differences $(P<0.05)$ as determined by a one-way ANOVA analysis followed by Tukey's HSD post hoc test. d, $\mathbf{g}$ Relative lesion length in $(\mathbf{b})$ and $(\mathbf{e})$, respectively. Error bars indicate SD $(n=3)$. Different letters above bars indicate significant differences $(P<0.05)$ as determined by a one-way ANOVA analysis and Tukey's HSD post hoc test

hpi (Fig. S1f); it was lower in STTM159 than in the control at 0, 12, and $24 \mathrm{hpi}$ (Fig. S2g). The expression of OsJA$M Y B$ was higher in OX159 than in the control at 6 and 12 hpi (Fig. S1g), while it was lower in STTM159 than in the control at 6 and 12 hpi (Fig. S2h). These data indicated that Osa-miR159a activated defense-related genes, positively regulating rice resistance to $M$. oryzae.

\section{Alteration of Osa-miR159a Accumulation Leads to Developmental Defects}

In addition to the resistance conferred by Osa-miR159a in rice against $M$. oryzae, we found that both OX159 and STTM159 showed some altered development and yield traits. All the OX159 and STTM159 transgenic lines were shorter than the control (Fig. 3a, b and Table 1), with STTM159 lines significantly shorter than OX159 lines and the control (Fig. 3b and Table 1). Both OX159 and STTM159 had a lower yield (Table 1). The OX159 lines were sterile and had only a few filled grains on the panicle, leading to straight panicles at the mature stage (Fig. 3a, c and Table 1). The stamen development was deficient in OX159 lines (Fig. 3e, f). In comparison with the control, which had yellowish anthers containing fertile pollen indicated by starch-staining, anthers from 


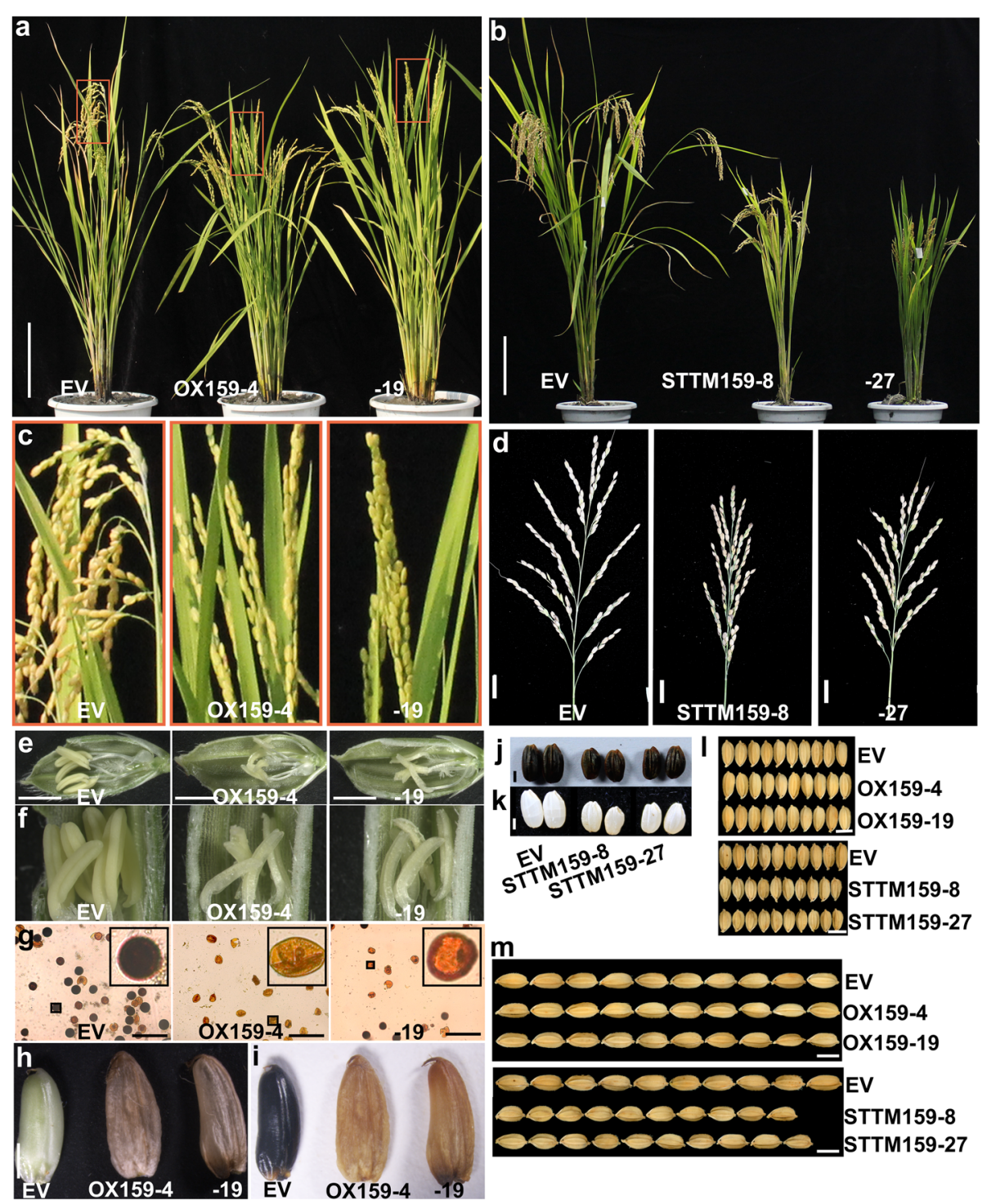

Fig. 3 Osa-miR159a influences rice traits and yield. a, b Gross morphology of the indicated lines. Scale bars, $20 \mathrm{~cm}$. c, d Panicles morphology of the indicated lines. Scale bars, $2 \mathrm{~cm}$ in (d). e, $\mathbf{f}$ Shucked grains of the indicated lines. Scale bars, $2 \mathrm{~mm}$. $\mathbf{g}$ Potassium iodide dye shows the starch in the pollen grain of the indicated lines. Scale bars, $100 \mu \mathrm{m}$. $\mathbf{h}-\mathbf{k}$ Potassium iodide dye shows the starch in seeds of the indicated lines. The pictures in $(\mathbf{h}, \mathbf{k})$ were captured before potassium iodide dye. The pictures in $(\mathbf{i}, \mathbf{j})$ were captured after potassium iodide dye. $\mathbf{I}, \mathbf{m}$ Comparison of grain width (I) and grain length $(\mathbf{m})$ in the indicated lines. Scale bars, $5 \mathrm{~mm}$

Table 1 Yield traits of the wild type, OX159, and STTM159 lines grown in rice paddies

\begin{tabular}{lllllllll}
\hline Materials & $\begin{array}{l}\text { Plant } \\
\text { Height/cm }\end{array}$ & $\begin{array}{l}\text { No. of } \\
\text { Tillers }\end{array}$ & $\begin{array}{l}\text { Panicle } \\
\text { Length/cm }\end{array}$ & $\begin{array}{l}\text { No. of Filled Gains } \\
\text { Per Plant }\end{array}$ & $\begin{array}{l}\text { Yield Per } \\
\text { Plant/g }\end{array}$ & $\begin{array}{l}\text { 1000-grain } \\
\text { weight/g }\end{array}$ & $\begin{array}{l}\text { Grain } \\
\text { Length/mm }\end{array}$ & $\begin{array}{l}\text { Grain Width/ } \\
\text { mm }\end{array}$ \\
\hline EV & $95.83 \pm 0.58^{\mathrm{a}}$ & $12.80 \pm 1.73^{\mathrm{a}}$ & $19.40 \pm 0.25^{\mathrm{a}}$ & $1096.33 \pm 23.50^{\mathrm{a}}$ & $28.26 \pm 0.94^{\mathrm{a}}$ & $25.76 \pm 0.49^{\mathrm{a}}$ & $7.19 \pm 0.013^{\mathrm{b}}$ & $3.32 \pm 0.02^{\mathrm{a}}$ \\
OX159-4 & $85.33 \pm 2.08^{\mathrm{b}}$ & $12.67 \pm 1.53^{\mathrm{a}}$ & $17.65 \pm 0.35^{\mathrm{b}}$ & $146.67 \pm 106.00^{\mathrm{d}}$ & $6.79 \pm 3.53^{\mathrm{d}}$ & $24.30 \pm 0.53^{\mathrm{b}}$ & $7.42 \pm 0.082^{\mathrm{a}}$ & $3.32 \pm 0.02^{\mathrm{a}}$ \\
OX159-19 & $87.00 \pm 1.00^{\mathrm{b}}$ & $12.00 \pm 2.65^{\mathrm{a}}$ & $17.46 \pm 0.30^{\mathrm{b}}$ & $34.00 \pm 28.48^{\mathrm{e}}$ & $0.82 \pm 0.68^{\mathrm{e}}$ & $24.07 \pm 0.13^{\mathrm{b}}$ & $7.46 \pm 0.050^{\mathrm{a}}$ & $3.32 \pm 0.012^{\mathrm{a}}$ \\
STTM159- & $58.96 \pm 5.63^{\mathrm{c}}$ & $8.20 \pm 1.30^{\mathrm{b}}$ & $15.74 \pm 0.38^{\mathrm{c}}$ & $670.00 \pm 22.30^{\mathrm{c}}$ & $12.70 \pm 0.60^{\mathrm{c}}$ & $18.97 \pm 0.15^{\mathrm{d}}$ & $6.38 \pm 0.15^{\mathrm{c}}$ & $3.34 \pm 0.0074^{\mathrm{a}}$ \\
8 & & & & & & & & \\
STTM159- & $66.82 \pm 1.71^{\mathrm{c}}$ & $11.00 \pm 1.87^{\mathrm{a}}$ & $15.49 \pm 0.58^{\mathrm{c}}$ & $875.80 \pm 68.56^{\mathrm{b}}$ & $19.15 \pm 0.75^{\mathrm{b}}$ & $21.90 \pm 0.23^{\mathrm{c}}$ & $6.60 \pm 0.08^{\mathrm{c}}$ & $3.34 \pm 0.0017^{\mathrm{a}}$ \\
27 & & & & & & & & \\
\hline
\end{tabular}


OX159 were pale with sterile pollen lacking starch (Fig. $3 g)$. In addition, grains from OX159 lines lacked starch accumulation, although the ovary grew to a size comparable to that of the control (Fig. 3h, i, l, m and Table 1). However, STTM159 showed smaller panicles than that of the control, but the starch accumulation in the grain was normal (Fig. 3d, j, k). STTM159 was also observed to be less productive than the control (Table 1). The grain width of STTM159 was the same as the control, whereas the grain length was shorter than the control (Fig. 3l, $\mathrm{m}$ and Table $1)$. These results indicated that the alteration of OsamiR159a expression led to defects in growth and development, particularly in pollen and grain development.

\section{Alteration of Osa-miR159a Expression Impacts the Expression of its Target Genes}

Six Osa-miR159 loci in rice generate five mature isoforms that share 18 central nucleotides (Fig. S3a). Among them, Osa-miR159a/b targeted two confirmed genes, namely, OsGAMYB (LOC_Os01g59660) and OsGAMYBL (LOC_Os06g40330) (Li et al. 2016a), and one predicated gene, LOC_Os10g05230 (encoding a C3HC4-type domain-containing zinc finger protein, herein designated OsZF) (Khan et al. 2017). The target sites in OsGAMYB and OsGAMYBL were in the codon region, whereas the target site was in a $5^{\prime}$ untranslated region (UTR) of OsZF (Fig. S3b). To examine how the expression of these genes was impacted by the alteration of Osa-miR159a expression in OX159 and STTM159, we performed a RT-qPCR analysis. As expected, the expression of all three genes was significantly less in OX159 than in the control (Fig. S3c). In contrast, the expression of all these genes was more in STTM159 than in the control (Fig. S3c). These data indicated that the overexpression of Osa-miR159a significantly suppressed the expression of its target genes, and the STTM of OsamiR159a prevented the suppression of Osa-miR159a on the expression of its target genes.
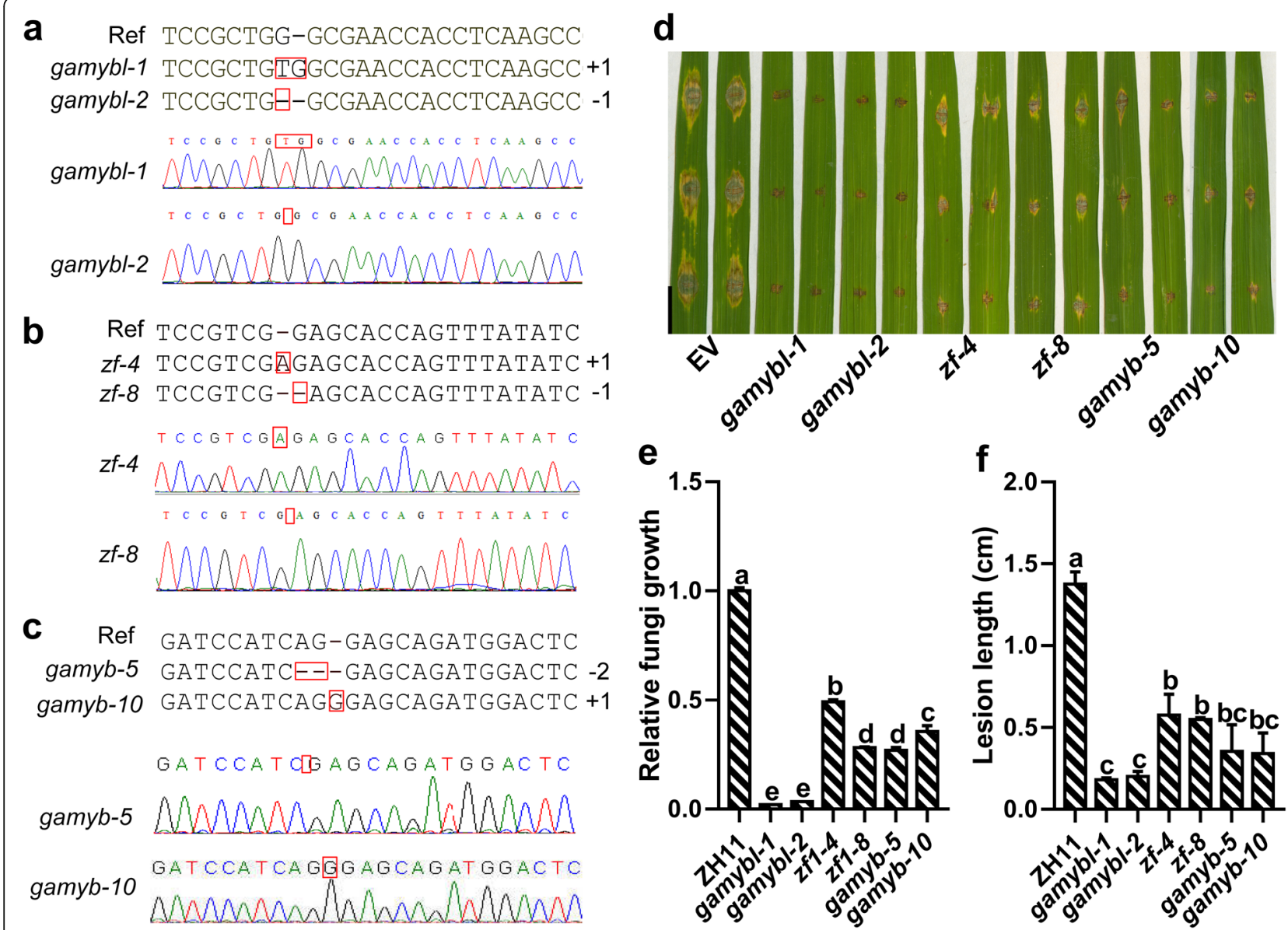

Fig. 4 The mutations of Osa-miR159a target genes result in enhanced resistance against Magnaporthe oryzae. a-c The genotype of Osa-miR159a target gene knockout lines were confirmed by PCR based sequencing. Ref means reference sequences. $\mathbf{d}$ Blast disease phenotype at 5 days postinoculation (dpi) with M. oryzae strain GZ8 in the indicated lines. Scale bars, $10 \mathrm{~mm}$. e, f Relative fungal biomass (e) and lesion length (f) on the inoculated leaves from $(\mathbf{d})$. Data are shown as mean \pm SD $(n=3)$. Different letters above bars indicate significant differences $(P<0.05)$ as determined by a one-way ANOVA and Tukey's HSD post hoc test. The experiments were repeated two times with similar results 


\section{Knockout of OsGAMYB, OsGAMYBL, and OsZF Leads to Enhanced Resistance to $M$. oryzae}

To investigate the function of OsGAMYB, OsGAMYBL, and OsZF, we obtained mutants using CRISPR/Cas9 DNA editing. We identified two independent mutants for OsGAMYBL, OsZF, and OsGAMYB. Among them, gamybl-1 carried a 1-bp insertion resulting in an early stop codon after aa 325 (Fig. 4a). gamybl-2 carried a 1bp deletion resulting in an early stop codon after aa 311 (Fig. 4a); $z f-4$ carried a 1-bp insertion resulting in an early stop codon after aa 42 (Fig. 4b). The $z f-8$ had a 1bp deletion resulting in an early stop code after aa 32 (Fig. 4b); gamyb-5 carried a 2-bp deletion resulting in an early stop codon after aa 127 (Fig. 4c). The gamyb-10 had a 1-bp insertion resulting in an early stop codon after aa 128 (Fig. 4c). We conducted a M. oryzae assay via punch-inoculation. All the knockout lines significantly decreased the size of $M$. oryzae lesions that contained significantly lower fungal mass and shorter lesions than that of the control (Fig. 4d-f). Specifically, gamybl mutants were especially resistant than gamyb and $z f$ mutants, indicating that OsGAMYBL has the most important role in disease resistance. Together, these results indicated that OsGAMYB, OsGAMYBL, and OsZF contributed to Osa-miR159a-mediated regulation of rice resistance to $M$. oryzae. However, based on previous studies, loss of function mutations of OsGAMYB resulted in shortened internodes and defects in floral organ development (Kaneko et al. 2004); loss function of OsGAMYBL also contribute to these phenotypes (Tsuji et al. 2006). Similarly, we found that loss of function mutations of OsGAMYB or OsGAMYBL cause defective rice development (data not shown), especially in relation to pollen development. However, the phenotype of $z f$ mutants was very similar to that of the wild type, except for the slightly reduced height of the $z f$ mutants (Fig. S4a), but not in 1000-grain weight (Fig. S4b). Together with the resistance phenotype, the editing of OsZF may have a potential application in rice breeding.

\section{Discussion}

miRNAs act as important regulators in plant growth, development, and host-pathogen interactions (JonesRhoades et al. 2006; Baldrich and San Segundo 2016). Some miRNAs have been identified to be involved in fine-tuning rice resistance to $M$. oryzae and yield traits. For example, high accumulation of Osa-miR1873 results in defects in growth and yield-related traits, and also increases susceptibility to M. oryzae (Zhou et al. 2020). Here, we described Osa-miR159a, which regulates multiple growth and yield traits, as a new positive regulator in rice resistance to $M$. oryzae. First, high accumulation of Osa-miR159a resulted in enhanced resistance to $M$. oryzae, which was associated with an increase in the defense response, i.e., high expression of defense-related genes (Fig. 2b-d and Fig. S1). In addition, the transgenic lines overexpressing Osa-miR159a showed developmental defects such as pollen sterility and grain-filling (Fig. 3a, c). However, blocking Osa-miR159a by STTM resulted in increased susceptibility to M. oryzae (Fig. 2e-g and Fig. S2). Developmental defects were also observed in STTM159 transgenic lines including shorter plants and reduced grain length (Fig. 3b, d, m). Consistent with the $M$. oryzae disease phenotypes in OX159 and STTM159, the OsGAMYB, OsGAMYBL, and OsZF knockout lines exhibited enhanced resistance to $M$. oryzae (Fig. 4). Therefore, Osa-miR159a has multiple functions in rice resistance to $M$. oryzae and rice development.

miR159 is a conserved miRNA that represses the expression of GAMYB-like genes, which encode MYB domain transcription factors (Alonso-Peral et al. 2010). Proper expression of GAMYB and GAMYBL are important for rice development. GAMYB acts as a positive transcriptional regulator of GA-dependent $\alpha$-amylase expression and also has important roles in floral organ development and pollen development (Kaneko et al. 2004). Here, we demonstrated that suppressing GAMYB by overexpressing Osa-miR159a resulted in sterile pollen lacking starch and failure of the grain to accumulate starch (Fig. 3e-i); however, the grain length was slightly larger than that of the wild type (Table 1). In addition, the uninhibited expression of GAMYB by overexpressing STTM159 also resulted in slightly shorter grains (Fig. $3 \mathrm{~m}$ and Table 1). These results indicated that GAMYB was crucial for grain development. We observed that enhanced or decreased expression of Osa-miR159a also impacted plant height (Fig. 3a, b and Table 1). Previous studies reported that loss of function mutations of OsGAMYB results in shortened internodes; OsGAMYBL contributes to this phenotype (Kaneko et al. 2004; Tsuji et al. 2006), indicating that OsGAMYB and OsGAMYBL played a role in the height of rice plants. However, $z f$ mutants showed slightly reduced height without other defects in rice development. Therefore, future studies should consider the potential application of OsZF in rice breeding using the CRISPR/Cas9 system.

The circadian clock and abiotic stress conditions impact gene expressions in plants (Sugiyama et al. 2001; Matsuzaki et al. 2015). Thus, mock treatments were necessary in examining the expression of Osa-miR159a during $M$. oryzae infection. The results showed that Osa-miR159a was responsive to $M$. oryzae infection compared with the mock treatment at each time point (Fig. 1). However, the mock treatment strongly influences the expression of Osa-miR159a, indicating that the expression of Osa-miR159a may be strongly impacted by the circadian clock or/and abiotic stress 
conditions (i.e., mock treatment), but further studies are required. In addition, our results showed that knockout lines of OsGAMYB and OsGAMYBL exhibited increased resistance to M. oryzae (Fig. 4). It is important to determine how Osa-miR159a functions in rice immunity against $M$. oryzae. On one hand, this is consistent with previous results that have shown that MYB transcription factors are involved in rice immunity. For example, the repressive MYB transcription factor, MYBS1, results in reduced expression of broad-spectrum resistance-Digu 1 (bsr-d1) allele from the rice cultivar Digu, thus, inhibiting $\mathrm{H}_{2} \mathrm{O}_{2}$ degradation and enhanced disease resistance ( $\mathrm{Li}$ et al. 2017b). MYB30 bonds to and activates the promoter of the 4-coumarate:coenzyme A ligase genes Os4CL3 and Os4CL5, resulting in accumulation of lignin subunits $\mathrm{G}$ and $\mathrm{S}$, further leading to obvious thickening of sclerenchyma cells and inhibiting $M$. oryzae penetration ( $\mathrm{Li}$ et al. 2020b). BGIOSGA004670, the homolog of GAMYB in Arabidopsis, showed increased expression upon fungal infection, suggesting that GAMYB-like genes might be involved in resistance to fungal infection ( $\mathrm{Li}$ et al. 2016b). The novel MYB transcription factor CaPHL8 acts as a positive regulator in the resistance of pepper to Ralstonia solanacerum (Noman et al. 2019). On the other hand, GAMYB is involved in GA-signaling; thus, GAMYB may regulate rice immunity by manipulating plant hormones. Moreover, loss-of-function of $Z F$ also results in enhanced resistance to M. oryzae. Zinc finger proteins are involved in plant growth and development. Overexpression of zinc finger protein 1 (GhZFP1) enhances resistance to Rhizoctonia solani (Guo et al. 2009). In the future, we will focus on the function of $Z F$ in the resistance of rice immunity to $M$. oryzae.

An increasing number of studies have shown that the production of RNAi-inducing dsRNA in the host can result in specific fungal gene silencing, further conferring resistance to fungal pathogens (Zhang et al. 2016b). In response to Verticillium dahlia infection, cotton plants increase miR166 and miR159 expression, and export them to fungal hyphae for silencing key genes that are essential for fungal virulence (Zhang et al. 2016b). Hence, we cannot exclude the possibility that OsamiR159a may also be exported to the fungal hyphae of $M$. oryzae to silence genes that are essential for fungal virulence. Future studies are required to confirm this hypothesis.

\section{Conclusions}

We functionally characterized Osa-miR159a and its target genes in rice resistance to $M$. oryzae. Our data indicated that Osa-miR159a positively regulated resistance to $M$. oryzae and impacted yield traits by regulating its target genes OsGAMYB, OsGAMYBL, and OsZF. Suppressed expression of OsGAMYB, OsGAMYBL, and OsZF by overexpressing Osa-miR159a or knockout of
OsGAMYB, OsGAMYBL, and OsZF resulted in enhanced resistance to $M$. oryzae, but led to developmental defects. In contrast, blocking Osa-miR159a via STTM led to significantly increased susceptibility and defects in yield traits. Therefore, proper spatiotemporal expression of Osa-miR159a was critical for rice immunity and development. Osa-miR159a and the regulatory module of its target genes could be used to breed rice with resistance to M. oryzae.

\section{Materials and Methods \\ Plant Materials and Growth Conditions}

Oryza sativa Japonica accessions Nipponbare (NPB) and Zhong Hua 11 (ZH11) were used for transgenic analysis. The susceptible accession Lijiangxin Tuan Heigu (LTH) and the resistant accession International Rice Blast Line Pyricularia-Kanto51-m-Tsuyuake (IRBLkm-Ts) was used in this study. The rice plants were grown in a growth chamber maintained at $26^{\circ} \mathrm{C}$ and $70 \%$ relative humidity under $12 \mathrm{~h}$ of light and $12 \mathrm{~h}$ of darkness. To assay yield traits, rice plants were grown in a paddy field in the Wenjiang district of Chengdu, China, from April to September.

\section{Analysis of Yield Traits}

Rice agronomic traits were measured from five plants in the paddy field at maturity. The 1000-grain weight, grain length, and grain width were measured using an SC-A grain analysis system (Wanshen Ltd., Hangzhou, China) using the filled grains that were dried in an oven at $42^{\circ} \mathrm{C}$ for 1 week. These data were analyzed by a one-way ANOVA followed by post hoc Tukey's HSD analysis. Differences were considered significant at $P<0.01$.

\section{Plasmid Construction and Genetic Transformation}

To construct the transgenic line overexpressing OsamiR159a, we amplified the DNA sequence containing 321 bp upstream and 306 bp downstream of Osa-miR159a from NPB genomic DNA with the specific primers miR159aKpnI-F and miR159a-SalI-R (Table S1), then the amplified DNA fragments were digested and cloned into the binary vector $35 \mathrm{~S}$-pCAMBIA1300 at KpnI and SalI sites, resulting in the overexpressing construct. To construct the transgenic lines overexpressing the short tandem target mimic (STTM) of Osa-miR159a (STTM159), we inserted the amplified DNA fragments of STTM159 into the KpnI and SalI sites of the binary vector 35S-pCAMBIA1300, resulting in a target mimic of the miR159 construct. The entire sequences of STTM159 were 5' -ggtaccTGCAGCTCCTGATCGGGCATGCAAGTTGTTGTTGTTATGGTCTAGTTGTTGTTGTTATGGTCTAATTTAAATA TGGTCTAAAGAAGAAGAATATGGTCTAAAGAA GAAGAATCAGAGCTCCCTCAGTCAATCCAA 
Agtcgac-3'. Both of the constructs were transformed into the NPB background via Agrobacterium-mediated transformation. To generate Osa-miR159a target gene knockout lines, we constructed the CRISPR/Cas9 plasmids as described previously (Zhao et al. 2019). The constructs were transformed into the ZH11 background via Agrobacterium strain GV3101. All the positive transgenic lines were confirmed using hygromycin. To confirm the genotype of the knockout lines, we performed PCR-based gene sequencing as described previously (Zhao et al. 2019). All primers are listed in Table S1.

\section{Pathogen Infection and Microscopy Analysis}

Magnaporthe oryzae strains Guy11 and eGFP-tagged Zhong10-8-14 (GZ8) was used in this study. The strain was incubated on oatmeal and tomato agar media (OTA) at $28^{\circ} \mathrm{C}$ under a 12-h light and 12-h dark cycle. After 10 days, the hyphae were scratched, and the fungus on the plates was further incubated with 24-h light treatment to promote sporulation. Three to 5 days later, the spores were collected for spray- or punch-inoculation. For spray-inoculation, seedlings at the three-leaf-stage were inoculated with a spore suspension $\left(3 \times 10^{5}\right.$ conidia $/ \mathrm{mL}$ ). Disease lesions were recorded at 5 days postinoculation (dpi). For punch-inoculation, $5 \mu \mathrm{L}$ of the spore suspension $\left(5 \times 10^{5}\right.$ spore/mL $)$ was dropinoculated at wound sites on the leaves of seedlings at the three-leaf-stage following previously described methods ( $\mathrm{Li}$ et al. 2014). Briefly, the dilution-drop conidia suspension was placed against wounded sites on the leaves. Lesion formation was examined at 4-6 dpi. The relative fungal mass was calculated using the DNA concentration of $M$. oryzae Pot 2 against the rice genomic Ubiquitin DNA level by quantitative PCR.

$M$. oryzae strain GZ8 was used to observe the fungal infection process. Leaf sheaths (5-cm-long) were inoculated with a spore suspension $\left(1 \times 10^{4}\right.$ conidia/mL) as described previously (Li et al. 2014). The inoculated epidermal layer was excised for observation. We observed the invasion process including appressorium development and invasive hyphal growth with a Laser Scanning Confocal Microscope (Nikon A1) at 24-, 36-, and 48hpi. The quantitative analysis of the infestation stage was conducted as described previously (Li et al. 2017a).

\section{Reverse Transcription Quantitative Polymerase Chain Reaction (RT-qPCR)}

The leaves of OX159 and STTM159 were collected to detect the amounts of miRNA and target genes. To examine the expressions of defense-related genes, we inoculated seedlings in the three-leaf-stage with $M$. oryzae using the spray inoculation method. The inoculated leaf samples were collected at $0,6,12$, and 24 hpi. Total
RNA was extracted, then reverse transcription was performed following a previous report (Zhao et al. 2021). To analyze the expression of miRNA, we performed a stem-loop pulse RT-qPCR following a previous report (Turner et al. 2013). U6 snRNA was used as an internal reference to normalize data.

\section{Abbreviations}

STTM: Short tandem target mimic; ARF: Auxin response factor; LTH: Lijiangxin Tuan Heigu; IRBLkm-Ts: International Rice Blast Line Pyricularia-Kanto51-mTsuyuake; NPB: Nipponbare; ZH11: Zhong Hua 11; CRISPR: Clustered regularly interspaced short palindromic repeats; OTA: Tomato agar media; RTqPCR: Reverse transcription quantitative polymerase chain reaction; LSCM: Laser scanning confocal microscopy; TCP: PROLIFERATING CELL NUCLEAR ANTIGEN BINDING FACTOR

\section{Supplementary Information}

The online version contains supplementary material available at https://doi. org/10.1186/s12284-021-00469-w.

Additional file 1 : Figure S1. Overexpression of Osa-miR159a results in enhanced resistance to Magnaporthe oryzae. a Blast disease phenotype at 5 days post-inoculation (dpi) with M. oryzae strain GZ8. Scale bars, $10 \mathrm{~mm}$. b Relative lesion area on the inoculated leaves from (a). Error bars indicate standard deviation (SD) $(n=3)$. Asterisks $\left(^{* *}\right)$ above the bar indicates significant differences $(P<0.01)$ determined by the Student's $t$-test. c Confocal images showing the infection status of eGFP-tagged $M$. oryzae strain GZ8 in the indicated lines at 24, 36, and $48 \mathrm{~h}$ post-inoculation (hpi). Scale bars, $25 \mu \mathrm{m}$. d Quantification analysis on the process of GZ8 infection in the indicated lines at the indicated time points. Over 200 conidia in each line were analyzed. The experiments were repeated two times with similar results. e-g The expression of defense-related genes in wild type and OX159 lines following the inoculation of M. oryzae strain GZ8. RNA was extracted at the indicated time points for the reverse transcriptionquantitative PCR (RT-qPCR) assay. The amounts of collected mRNA were normalized to that in the wild type at 0 hpi. Error bars indicate SD $(n=3)$. Different letters above the bars indicate significant differences $(P<0.01)$ as determined by a one-way ANOVA followed by post hoc Tukey's HSD analysis.

Additional file $\mathbf{2}$ : Figure S2. Overexpression of STTM159 results in enhanced susceptibility to Magnaporthe oryzae. a Blast disease phenotype at 5 days post-inoculation (dpi) with M. oryzae strain GZ8. Scale bars, $10 \mathrm{~mm}$. b. c Relative fungal growth and lesion area on the inoculated leaves from $(\mathbf{a})$. Error bars indicate SD $(n=3)$. Asterisks $\left(^{* *}\right)$ above the bar indicates significant differences $(P<0.01)$ determined by the Student's $t$-test. $\mathbf{d}$ Confocal images showing the infection status of a GFPtagged M. oryzae strain GZ8 in the indicated lines at 24,36, and $48 \mathrm{~h}$ post-inoculation (hpi). Scale bars, $25 \mu \mathrm{m}$. e Quantification analysis of the process of GZ8 infection in the indicated lines at the indicated time points. Over 200 conidia in each line were analyzed. The experiments were repeated two times with similar results. $\mathbf{f}-\mathbf{h}$ The expression of defense-related genes in wild type (WT) and STTM159 lines following the inoculation of $M$. oryzae strain GZ8. RNA was extracted at the indicated time points for the reverse transcription-quantitative PCR (RT-qPCR) assay. The amounts of collected mRNA were normalized to that in the WT at 0 hpi. Error bars indicate SD $(n=3)$. Different letters above the bars indicate significant differences $(P<0.01)$ as determined by a one-way ANOVA followed by post hoc Tukey's HSD analysis.

Additional file 3 : Figure S3. Osa-miR159 mature isoforms and the accumulation of Osa-miR159a target genes in the indicated lines. a The sequence alignments of Osa-miR159 mature isoforms and their positions on the chromosome in rice. $\mathbf{b}$ The structure of target genes and the sequence alignment of the target sites in the target genes. White boxes indicate the 5'-UTRs and 3'-UTRs. Black boxes indicate exons. Black lines indicate introns. Red lines indicate the target sites of Osa-miR159a. c Reverse transcription-quantitative PCR (RT-qPCR) data show the relative mRNA amount of target genes in OX159 and STTM159 in comparison 
with NPB containing the empty vector (EV). Data are shown as mean \pm SD $(n=3)$. Different letters above bars indicate significant differences $(P<$ 0.05 ) as determined by a one-way ANOVA followed by post hoc Tukey's HSD analysis.

Additional file 4 : Figure S4. The phenotype and 1000-grain weight (g) of the zf mutants. a The phenotype of zf mutants and EV control at the reproductive stage. The height of zf mutants slightly less than that of the wild type plants. b The 1000-grain weight (g) of the indicated plants showed no difference.

Additional file $\mathbf{5}$ : Table S1. The primers used in this research.

\section{Acknowledgments}

We thank Sichuan Applied Fundamental Research Foundation and the National Natural Science Foundation of China for funding.

\section{Authors' Contributions}

J.-F.C. performed the experiments with support from Z.-X.Z., Y.L., T.-T.L., Y.Z., X.-M.Y., S.-X.Z., H.W., J.-Q.Z., M.P., H.F., J.F., J.-W.Z., and Y.-Y.H. W.-M.W. conceived the project and designed the experiments. Z.-X.Z., J.-F.C., and W.M.W. analyzed the data; Z.-X.Z., Y.L. and W.-M.W. wrote the manuscript. J.-F.C. Z.-X.Z. and Y.L. contributed equally to this work. The author(s) read and approved the final manuscript.

\section{Funding}

This work was supported by the Sichuan Applied Fundamental Research Foundation (2020YJ0332) and the National Natural Science Foundation of China (U19A2033, 31672090, and 31430072) to W-M.W.

\section{Availability of Data and Materials}

All of the datasets are included within the article and its additional files.

\section{Declarations}

\section{Ethics Approval and Consent to Participate}

Not applicable.

\section{Consent for Publication}

Not applicable.

\section{Competing Interests}

All the authors declare no conflict of interests.

\section{Received: 27 August 2020 Accepted: 24 February 2021}

Published online: 06 March 2021

\section{References}

Achard P, Herr A, Baulcombe DC, Harberd NP (2004) Modulation of floral development by a gibberellin-regulated microRNA. Development 131:33573365

Allen RS, Li J, Stahle MI, Dubroué A, Gubler F, Millar AA (2007) Genetic analysis reveals functional redundancy and the major target genes of the Arabidopsis miR159 family. Proc Natl Acad Sci U S A 104:16371-16376

Alonso-Peral MM, Li J, Li Y, Allen RS, Schnippenkoetter W, Ohms S, White RG, Millar AA (2010) The MicroRNA159-regulated GAMYB-like genes inhibit growth and promote programmed cell death in Arabidopsis. Plant Physiol 154:757-771

Baldrich P, San Segundo B (2016) MicroRNAs in rice innate immunity. Rice 9:6

Chandran V, Wang H, Gao F, Cao X-L, Chen Y-P, Li G-B, Zhu Y, Yang X-M, Zhang L-L, Zhao Z-X, Zhao J-H, Wang Y-G, Li S, Fan J, Li Y, Zhao J-Q, Li S-Q, Wang W-M (2019) miR396-OsGRFs module balances growth and rice blast diseaseresistance. Front Plant Sci 9:1999

Franco-Zorrilla JM, Valli A, Todesco M, Mateos I, Puga MI, Rubio-Somoza I, Leyva A, Weigel D, García JA, Paz-Ares J (2007) Target mimicry provides a new mechanism for regulation of microRNA activity. Nat Genet 39:1033-1037

Gao X, Zhang Q, Zhao Y-Q, Yang J, He H-B, Jia G-X (2020) The Ire-miR159aLrGAMYB pathway mediates resistance to grey mould infection in Lilium regale. Mol Plant Pathol 21:749-760

Guo C, Xu Y, Shi M, Lai Y, Wu X, Wang H, Zhu Z, Poethig RS, Wu G (2017) Repression of miR156 by miR159 regulates the timing of the juvenile-toadult transition in Arabidopsis. Plant Cell 29:1293-1304
Guo Y-H, Yu Y-P, Wang D, Wu C-A, Yang G-D, Huang J-G, Zheng C-C (2009) GhZFP1, a novel CCCH-type zinc finger protein from cotton, enhances salt stress tolerance and fungal disease resistance in transgenic tobacco by interacting with GZIRD21A and GZIPR5. New Phytol 183:62-75

Jones-Rhoades MW, Bartel DP, Bartel B (2006) MicroRNAs and their regulatory roles in plants. Annu Rev Plant Biol 57:19-53

Kaneko M, Inukai Y, Ueguchi-Tanaka M, Itoh H, Izawa T, Kobayashi Y, Hattori T, Miyao A, Hirochika H, Ashikari M, Matsuoka M (2004) Loss-of-function mutations of the rice GAMYB gene impair a-amylase expression in aleurone and flower development. Plant Cell 16:33-44

Khan A, Goswami K, Sopory SK, Sanan-Mishra N (2017) "Mirador" on the potential role of miRNAs in synergy of light and heat networks. Indian J Plant Physiol 22:587-607

Li H, Hu B, Wang W, Zhang Z, Liang Y, Gao X, Li P, Liu Y, Zhang L, Chu C (2016a) Identification of microRNAs in rice root in response to nitrate and ammonium. J Genet Genomics 43:651-661

Li W, Wang K, Chern M, Liu Y, Zhu Z, Liu J, Zhu X, Yin J, Ran L, Xiong J, He K, Xu L, He M, Wang J, Liu J, Bi Y, Qing H, Li M, Hu K, Song L, Wang L, Qi T, Hou Q, Chen W, Li Y, Wang W, Chen X (2020b) Sclerenchyma cell thickening through enhanced lignification induced by OsMYB30 prevents fungal penetration of rice leaves. New Phytol 226:1850-1863

Li W, Zhu Z, Chern M, Yin J, Yang C, Ran L, Cheng M, He M, Wang K, Wang J, Zhou X, Zhu X, Chen Z, Wang J, Zhao W, Ma B, Qin P, Chen W, Wang Y, Liu J, Wang W, Wu X, Li P, Wang J, Zhu L, Li S, Chen X (2017b) A natural allele of a transcription factor in rice confers broad-spectrum blast resistance. Cell 170:114-126.e115

Li X-P, Ma X-C, Wang H, Zhu Y, Liu X-X, Li T-T, Zheng Y-P, Zhao J-Q, Zhang J-W, Huang Y-Y, Pu M, Feng H, Fan J, Li Y, Wang W-M (2020a) Osa-miR162a finetunes rice resistance to Magnaporthe oryzae and yield. Rice 13:38

Li Y, Cao X-L, Zhu Y, Yang X-M, Zhang K-N, Xiao Z-Y, Wang H, Zhao J-H, Zhang LL, Li G-B, Zheng Y-P, Fan J, Wang J, Chen X-Q, Wu X-J, Zhao J-Q, Dong OX, Chen X-W, Chern M, Wang W-M (2019a) Osa-miR398b boosts $\mathrm{H}_{2} \mathrm{O}_{2}$ production and rice blast disease-resistance via multiple superoxide dismutases. New Phytol 222:1507-1522

Li Y, Jeyakumar JMJ, Feng Q, Zhao Z-X, Fan J, Khaskheli MI, Wang W-M (2019b) The roles of rice microRNAs in rice-Magnaporthe oryzae interaction. Phytopathol Res 1:33

Li Y, Lu Y-G, Shi Y, Wu L, Xu Y-J, Huang F, Guo X-Y, Zhang Y, Fan J, Zhao J-Q, Zhang H-Y, Xu P-Z, Zhou J-M, Wu X-J, Wang P-R, Wang W-M (2014) Multiple rice microRNAs are involved in immunity against the blast fungus Magnaporthe oryzae. Plant Physiol 164:1077-1092

Li Y, Zhao S-L, Li J-L, Hu X-H, Wang H, Cao X-L, Xu Y-J, Zhao Z-X, Xiao Z-Y, Yang N, Fan J, Huang F, Wang W-M (2017a) Osa-miR169 negatively regulates rice immunity against the blast fungus Magnaporthe oryzae. Front Plant Sci 8:2

Li Z-Y, Xia J, Chen Z, Yu Y, Li Q-F, Zhang Y-C, Zhang J-P, Wang C-Y, Zhu X-Y, Zhang W, Chen Y-Q (2016b) Large-scale rewiring of innate immunity circuitry and microRNA regulation during initial rice blast infection. Sci Rep 6:25493

Matsuzaki J, Kawahara Y, Izawa T (2015) Punctual transcriptional regulation by the rice circadian clock under fluctuating field conditions. Plant Cell 27:633-648

Miao C, Wang D, He R, Liu S, Zhu J-K (2020) Mutations in MIR396e and MIR396f increase grain size and modulate shoot architecture in rice. Plant Biotechnol J 18:491-501

Millar AA, Lohe A, Wong G (2019) Biology and function of miR159 in plants. Plants 8:255

Miura K, Ikeda M, Matsubara A, Song X-J, Ito M, Asano K, Matsuoka M, Kitano H, Ashikari M (2010) OsSPL14 promotes panicle branching and higher grain productivity in rice. Nat Genet 42:545-549

Noman A, Hussain A, Adnan M, Khan MI, Ashraf MF, Zainab M, Khan KA, Ghramh HA, He S (2019) A novel MYB transcription factor CaPHL8 provide clues about evolution of pepper immunity againstsoil borne pathogen. Microb Pathog 137:103758

Palatnik JF, Wollmann H, Schommer C, Schwab R, Boisbouvier J, Rodriguez R, Warthmann N, Allen E, Dezulian T, Huson D, Carrington James C, Weigel D (2007) Sequence and expression differences underlie functional specialization of Arabidopsis microRNAs miR159 and miR319. Dev Cell 13:115-125

Pan X, Li Y, Zhang H, Huang R, Liu W, Ming J, Liu S, Li X (2014) Expression of signalling and defence-related genes mediated by over-expression of JERF1, and increased resistance to sheath blight in rice. Plant Pathol 63:109-116

Park C-H, Chen S, Shirsekar G, Zhou B, Khang CH, Songkumarn P, Afzal AJ, Ning Y, Wang R, Bellizzi M, Valent B, Wang G-L (2012) The Magnaporthe oryzae effector AvrPiz-t targets the RING E3 ubiquitin ligase APIP6 to suppress 
pathogen-associated molecular pattern-triggered immunity in rice. Plant Cell 24:4748-4762

Quoc NB, Phuong NDN, Trang HTT, Phi NB, Chau NNB (2019) Expression of OsamiR7695 against the blast fungus Magnaporthe oryzae in Vietnamese rice cultivars. Eur J Plant Pathol 155:307-317

Salvador-Guirao R, Y-i H, San Segundo B (2018) The polycistronic miR166k-166h positively regulates rice immunity via post-transcriptional control of EIN2. Front Plant Sci 9:337

Schwab R, Palatnik JF, Riester M, Schommer C, Schmid M, Weigel D (2005) Specific effects of microRNAs on the plant transcriptome. Dev Cell 8:517-527

Song X, Li Y, Cao X, Qi Y (2019) MicroRNAs and their regulatory roles in plantenvironment interactions. Annu Rev Plant Biol 70:489-525

Sugiyama N, Izawa T, Oikawa T, Shimamoto K (2001) Light regulation of circadian clock-controlled gene expression in rice. Plant J 26:607-615

Sun M, Shen Y, Li H, Yang J, Cai X, Zheng G, Zhu Y, Jia B, Sun X (2019) The multiple roles of OsmiR535 in modulating plant height, panicle branching and grain shape. Plant Sci 283:60-69

Todesco M, Rubio-Somoza I, Paz-Ares J, Weigel D (2010) A collection of target mimics for comprehensive analysis of microRNA function in Arabidopsis thaliana. PLoS Genet 6:e1001031-e1001031

Tsuji H, Aya K, Ueguchi-Tanaka M, Shimada Y, Nakazono M, Watanabe R, Nishizawa NK, Gomi K, Shimada A, Kitano H, Ashikari M, Matsuoka M (2006) GAMYB controls different sets of genes and is differentially regulated by microRNA in aleurone cells and anthers. Plant J 47:427-444

Turner M, Adhikari S, Subramanian S (2013) Optimizing stem-loop qPCR assays through multiplexed cDNA synthesis of U6 and miRNAs. Plant Signal Behav 8:e24918

Wang Z, Xia Y, Lin S, Wang Y, Guo B, Song X, Ding S, Zheng L, Feng R, Chen S, Bao Y, Sheng C, Zhang X, Wu J, Niu D, Jin H, Zhao H (2018) Osa-miR164a targets OsNAC60 and negatively regulates rice immunity against the blast fungus Magnaporthe oryzae. Plant J 95:584-597

Wu M-F, Tian Q, Reed JW (2006) Arabidopsis microRNA167 controls patterns of ARF6 and ARF8 expression, and regulates both female and male reproduction. Development 133:4211-4218

Yan J, Gu Y, Jia X, Kang W, Pan S, Tang X, Chen X, Tang G (2012) Effective small RNA destruction by the expression of a short tandem target mimic in Arabidopsis. Plant Cell 24:415-427

Yan J, Zhao C, Zhou J, Yang Y, Wang P, Zhu X, Tang G, Bressan RA, Zhu J-K (2016) The miR165/166 mediated regulatory module plays critical roles in ABA homeostasis and response in Arabidopsis thaliana. PLoS Genet 12:e1006416

Zhang H, Hu J, Qian Q, Chen H, Jin J, Ding Y (2016a) Small RNA profiles of the rice PTGMS line Wuxiang $S$ reveal miRNAs involved in fertility transition. Front Plant Sci 7:514

Zhang J, Zhou Z, Bai J, Tao X, Wang L, Zhang H, Zhu J-K (2019) Disruption of MIR396e and MIR396f improves rice yield under nitrogen-deficient conditions. Natl Sci Rev 7:102-112

Zhang T, Zhao Y-L, Zhao J-H, Wang S, Jin Y, Chen Z-Q, Fang Y-Y, Hua C-L, Ding S-W, Guo H-S (2016b) Cotton plants export microRNAs to inhibit virulence gene expression in a fungal pathogen. Nat Plants 2:16153

Zhang X, Bao Y, Shan D, Wang Z, Song X, Wang Z, Wang J, He L, Wu L, Zhang Z, Niu D, Jin H, Zhao H (2018) Magnaporthe oryzae induces the expression of a microRNA to suppress the immune response in rice. Plant Physiol 177:352-368

Zhao Y, Wang S, Wu W, Li L, Jiang T, Zheng B (2018) Clearance of maternal barriers by paternal miR159 to initiate endosperm nuclear division in Arabidopsis. Nat Commun 9:5011

Zhao Z-X, Feng Q, Cao X-L, Zhu Y, Wang H, Chandran V, Fan J, Zhao J-Q, Pu M, Li Y, Wang W-M (2019) Osa-miR167d facilitates infection of Magnaporthe oryzae in rice. J Integr Plant Biol 62:702-715

Zhao Z-X, Feng Q, Liu P-Q, He X-R, Zhao J-H, Xu Y-J, Zhang L-L, Huang Y-Y, Zhao J-Q, Fan J, Li Y, Xiao S, Wang W-M (2021) RPW8.1 enhances the ethylene signaling pathway to feedback attenuate its mediated cell death and disease resistance in Arabidopsis. New Phytol 229:516-531

Zhou S-X, Zhu Y, Wang L-F, Zheng Y-P, Chen J-F, Li T-T, Yang X-M, Wang H, Li XP, Ma X-C, Zhao J-Q, Pu M, Feng H, Li Y, Fan J, Zhang J-W, Huang Y-Y, Wang W-M (2020) Osa-miR1873 fine-tunes rice immunity against Magnaporthe oryzae and yield traits. J Integr Plant Biol 62:1213-1226

\section{Publisher's Note}

Springer Nature remains neutral with regard to jurisdictional claims in published maps and institutional affiliations.

\section{Submit your manuscript to a SpringerOpen ${ }^{\circ}$ journal and benefit from:}

- Convenient online submission

- Rigorous peer review

- Open access: articles freely available online

- High visibility within the field

- Retaining the copyright to your article

Submit your next manuscript at $\boldsymbol{\sim}$ springeropen.com 